\title{
Identification of sustainable control and digital dimension errors in business organizations
}

\author{
Mikail Khudzhatov ${ }^{1, *}$, Zhanna Chupina ${ }^{1}$, Alexey Ukhanov ${ }^{1}$, Tamara Dakhilgova ${ }^{1}$, and \\ Aleksei Sorokin ${ }^{1}$ \\ ${ }^{1}$ RUDN University, 117198, Moscow, Miklukho-Maklaya str.6, Russia
}

\begin{abstract}
In recent years, the technique of centralized control of complex production processes has moved from the stage of development and experimentation to widespread industrial implementation. Centralized control equipment is used in large, complex objects, and the control systems themselves are so complex that they cannot be calculated or reasonably selected using the methods used for conventional control and measuring equipment. The work undertaken recently in order to create various calculation methods for centralized control systems, at best, solved individual particular problems, often from different initial positions, and, apparently, there was a need for generalizing work that would give the system designers centralized control is an efficient settlement tool. Of the technical characteristics of centralized control systems, the most essential and most fully reflecting the effectiveness of their operation is accuracy, especially if this concept is broadly approached, including any deviation from the required system behavior in the error. Centralized control systems are used in various fields: to control production processes, for scientific research, including space objects, for testing and researching technical structures, for monitoring the health of complex equipment. These applications have a number of similarities and, however, differ in the number of requirements put forward by individual applications. When writing the article, the main attention was paid to centralized control systems for continuous production processes, which, in contrast to a number of testing and research systems, are characterized by the duration of action, since the production processes themselves in most cases run continuously for many hours, days or even months.
\end{abstract}

\section{Introduction}

Before proceeding to the calculation of errors in the performance of certain operations of centralized control over data processing, as well as to the accuracy characteristics of systems, a number of questions should be clarified: the accuracy of which particular basic transformations should be evaluated; how can the accuracy of the centralized control system as a whole be characterized; what requirements should be placed on the assessment of accuracy (how to accurately estimate the error) [1].

\footnotetext{
*Correspondent author: vSe.1@mail.ru
} 
The purpose of those signal transformations that are carried out by the nodes of the centralized control system can be either measurement - an assessment of a certain investigated physical quantity in the units with which it is customary to characterize, or control - making a judgment about which of several of our pre-selected areas the controlled value belongs to. Taking into account that the measurement result is expressed in digital form, these operations differ only in the form of interpretation of the result, but precisely because of this difference, a different approach to assessing their accuracy is required [2]. On the other hand, the commonality that exists between digital measurement and control makes it possible, under certain conditions, to go from measurement error to control error and back.

The random nature of a number of error components, and in many cases also of controlled processes, makes a probabilistic approach to the assessment of measurement and control advisable [3]. When analyzing the relationship between the probabilistic characteristics of measurement and control errors and the economic indicators of the efficiency of the systems, recommendations are developed for the use in different cases of different probabilistic characteristics of errors to assess the accuracy of measurement and control operations [4]. When considering a stochastic approach to assessing centralized control systems, a reasonable accuracy of estimating measurement and control errors is characterized [5]. It also formulates the rules of addition or accumulation of errors introduced into the overall result at individual stages of signal conversion.

The operations of digital measurement and control of analog signals have the same physical nature and differ only in the interpretation and assessment of the results obtained [6]. In both operations, the analog signal is converted while maintaining its analog representation, and then, by comparison with some reference signals (comparison level), it is mapped to a finite number of intervals [7]. In digital measurement, a certain number of units of the measured value is assigned to each interval, while in control, they are limited to the designation of the interval by means of one or another symbol.

The physical quantity $u$ to be measured or monitored passes through an analog processing device (hereinafter referred to as AAD). The signal $\mathrm{x}$ at the output of the UAO is subjected to analog-to-digital conversion; an analog-to-digital converter (hereinafter referred to as ADC) produces a digital signal $\mathrm{z}$, which, having passed through a digital processing device (hereinafter referred to as an ADC), is converted into a digital signal y. Note that the digital signal $\mathrm{z}$ and $\mathrm{y}$ can be converted back to an analog signal; such a conversion is sometimes performed in automated control systems, but in automatic measurement and control systems it is disadvantageous and therefore usually not applied. Therefore, the typical circuit we are analyzing first converts the analog signal, then digitizes it, and finally processes the digital signal [8]. In a significant part of devices, there is no digital processing link (hereinafter referred to as UPC), so that the result of digital measurement or control is the direct result of analog-to-digital conversion.

The purpose of the analog processing performed by the AAD device is to convert the input quantity $\mathrm{u}$ into a quantity $\mathrm{x}$ suitable for performing analog-to-digital conversion on it. Usually, they strive to obtain a linear dependence of $\mathrm{x}$ on $\mathrm{u}$, but this is not of fundamental importance, since the linearization of the measurement can also be carried out in the process of analog-to-digital conversion, and for control it is not required at all.

\section{Problem Statement}

Systematization of errors as a process of transformation. Let us first consider the essence of analog-to-digital conversion on the assumption that it is performed by the ADC converter without any error. The transformation consists in the fact that any value of the transformed quantity x refers by the transformer to one of the intervals $\boldsymbol{Q}_{i}, \mathrm{i}=1, \ldots, \mathrm{n}$, so that the converter issues from its output the digital code of the $\mathrm{i}$-th interval. This digital code can be considered 
as the result of a control operation performed on the value of $x$, and by virtue of the assumption that the analog-to-digital conversion is error-free, the error probability in this case is zero.

Analog-to-digital conversion is performed by comparing the converted value $\mathrm{x}$ with a number of levels $S_{1}, \ldots, S_{j}$, and from the totality of the results of these comparisons it logically follows that the value x belongs to a certain interval $\boldsymbol{Q}_{\boldsymbol{i}}$, the boundaries of which are certain combinations of comparison levels $\boldsymbol{S}_{\boldsymbol{j}}$.

In contrast to the control operation, the digital measurement operation is always accompanied by a methodical error. When the measured value is referred by the analog-todigital converter to the interval $\boldsymbol{Q}_{\boldsymbol{k}}$, then it is assigned the value y $=\boldsymbol{x}_{\boldsymbol{k}}$, usually equal to the value that the converted value takes in the middle of the interval $\boldsymbol{Q}_{\boldsymbol{k}}$. Analog-to-digital conversion using the signal at the output of the converter as discrete analog is a non-linear functional conversion. This transformation is commonly referred to as level quantization. Level quantization error $\eta$ functionally depends on the value of the quantized quantity $\mathrm{x}$ and can be written as a sum over all intervals of digital transformation, where through $\Pi_{\mathbf{0}}(\boldsymbol{t})$ we denote the function of a single "window" described by the formula:

$$
\begin{gathered}
\Pi_{0}(t)=\left\{\begin{array}{c}
0, t<\frac{1}{2}, t>\frac{1}{2}, \\
1,-\frac{1}{2}<t<\frac{1}{2} .
\end{array}\right. \\
\eta=\sum_{k} x_{k} \Pi_{0}\left(\frac{x-x_{k}}{Q_{k}}\right)-x=\sum_{k}\left(x_{k}-x\right) \Pi\left(\frac{x-x_{k}}{Q_{k}}\right),
\end{gathered}
$$

Ratio could be directly used to calculate the digital measurement error if there were no hardware errors in the UAO and ADC converters, or the methodical conversion error in the UAO device. However, such errors do exist.

\section{Research Questions}

As noted earlier, one of the main functions of centralized control equipment is measurement. The issues of measurement accuracy have been developed for more than one century, and at present the section on accuracy - a very important section of the science of measurement metrology - has been deeply and carefully developed. Of course, a number of metrology provisions apply to the accuracy of centralized process control; however, the essential features of centralized control force to develop a new approach to assessing its accuracy. At the same time, it should be noted that when assessing the measurement accuracy, one can partially use the methods and results of the theory of automatic control. First of all, it should be pointed out that traditional metrology considers the measurement of a physical quantity, about which it is assumed that it remains constant for a certain period of time, at least until the end of a series of measurements, with the help of which the results of previous measurements are refined. Classical metrology did not deal with time-varying processes; Only in recent years, works on the accuracy of measuring processes in time began to appear [9]. Thus, the main question that is raised in the study is how to evaluate errors and control of measurement in business organizations.

\section{Purpose of the Study}

The article discusses the calculation of the accuracy characteristics of centralized control systems, and also considers the application of the probabilistic approach to the calculation of accuracy. Therefore, the article is recommended not only for engineers who work in the field 
of automation and instrumentation, but also for economists who can apply this knowledge in the work of business organizations.

\section{Research Methods}

Let us consider the steady state in which there is no dynamic error of the UAO converter. Then the difference between the comparison level $\boldsymbol{S}_{\boldsymbol{j}}$, expressed in units of measurement of the input quantity $u$, and the actual value $u$ at the moment when the ADC comparison unit gives a signal about their equality, $\varphi=\boldsymbol{S}_{\boldsymbol{j}}$-u will be called the reduced comparison error. This error is the sum of the conversion error $\mathrm{u}$ to $\mathrm{x}$ in comparison with the result obtained from the calculated functional dependence and the calibration error of the comparison levels $\boldsymbol{S}_{\boldsymbol{i}}$ (taking into account the error of the comparison scheme). In other words, under $\varphi$ means the hardware error of the UAO converter and the comparison device of the analog-to-digital converter of the ADC, reduced directly to the comparison device, to which, possibly, the methodical error of the functional conversion (if it is performed in the UAO) is added. Let us now turn to finding the probabilistic characteristics of control errors and digital measurements. Considering first Figure 1, which does not contain a digital processing device $(y=z)$, and then extend the results to the complete circuit.

As stated earlier, to fully characterize random control error is to determine the probabilities $\boldsymbol{p}_{\boldsymbol{i} \boldsymbol{k}}$ Twow, the quantity u, which is actually contained in the region $\boldsymbol{Q}_{\boldsymbol{k}}$, will be referred to the area $\boldsymbol{Q}_{\boldsymbol{i}}$. The technique of controlling the vector quantity u is reduced to comparing one scalar quantity with the threshold levels $\boldsymbol{S}_{\boldsymbol{j}}, \mathrm{j}=1, \ldots, \boldsymbol{n}-\mathbf{1}$, or $\boldsymbol{n}-\mathbf{1}$ scalar with one threshold. In the general case, control can be reduced to comparison $\boldsymbol{n}-\mathbf{1}$ scalar $\boldsymbol{f}_{\boldsymbol{k}}(\boldsymbol{u}), \mathrm{k}=1, \ldots, \boldsymbol{n}-\mathbf{1}$ with a zero threshold. Considering this circumstance, in the future, estimating the probability of a control error, we will confine ourselves to simply considering the scalar controlled quantity $\mathrm{u}$.

Let, on the basis of the design characteristics of the UAO and ADC devices or from their experimental study, we know the conditional distribution density $\omega_{\varphi}(\varphi \mid \boldsymbol{u})$ reduced comparison error $\varphi$ depending on the value taken by the input quantity $u$. Then we can find the conditional probability of assigning the quantity u to the interval $\boldsymbol{Q}_{\boldsymbol{i}}$. From the fact that $u$ belongs to the interval $\boldsymbol{Q}_{i}$ given that

$\boldsymbol{a}_{\boldsymbol{i}-1}<\boldsymbol{u}+\boldsymbol{\varphi} \leq \boldsymbol{a}_{\boldsymbol{i}}$

$$
a_{i-1}-u<\varphi \leq a_{i}-u,
$$

1. Where $\boldsymbol{a}_{\boldsymbol{i}-\mathbf{1}}$ и $\boldsymbol{a}_{\boldsymbol{i}}$ - respectively, the lower and upper boundaries of the interval $\boldsymbol{Q}_{\boldsymbol{i}}$, follows,

$$
\mathbf{p}_{\mathbf{i}}(\mathbf{u})=\int_{\mathbf{a}_{\mathbf{i}-1}}^{\mathbf{a}_{\mathbf{i}}} \omega_{\varphi}(\mathbf{x}-\mathbf{u} \mid \mathbf{u}) \mathbf{d x}
$$

Conditional probability $\boldsymbol{p}_{\boldsymbol{i}}(\mathrm{u})$ takes into account the random nature of the given comparison error alone $\boldsymbol{\psi}$. If we consider the controlled variable $U$ as random as well, then we can find the probability $\boldsymbol{p}_{\boldsymbol{i} \boldsymbol{k}}$ control errors $\boldsymbol{\varepsilon}_{\mathbf{i k}}$ as:

$\mathbf{p}_{\mathrm{ik}}=\int_{\mathbf{a}_{\mathbf{k}-1}}^{\mathrm{a}_{\mathbf{k}}} \mathbf{p}_{\mathbf{i}}(\mathbf{u}) \boldsymbol{\omega}_{\mathbf{u}}(\mathbf{u}) \mathbf{d u},(5)$

Where $\boldsymbol{\omega}_{\mathbf{u}}(\mathbf{u})$ - probability distribution density of the controlled quantity $U$.

Let's deal with the measurement error further $\mathrm{E}$, for which you need to find the distribution density conditional $\boldsymbol{\omega}_{\boldsymbol{u}}(\mathcal{E} \mid \mathrm{u})$ and unconditional $\boldsymbol{\omega}_{\boldsymbol{u}}(\mathcal{E})$. Reference condition $\boldsymbol{x}_{\boldsymbol{i}}$ expressed by inequality $\boldsymbol{a}_{\boldsymbol{i}-\mathbf{1}}-\boldsymbol{u}<\boldsymbol{\varphi} \leq \boldsymbol{a}_{\boldsymbol{i}}-\boldsymbol{u}$, whence we have for the conditional distribution of the error $\mathrm{E}$ 
$\boldsymbol{\omega}_{\mathbf{u}}(\boldsymbol{\varepsilon} \mid \mathbf{u})=\sum_{\mathbf{i}} \mathbf{p}_{\mathbf{i}}(\mathbf{u}) \boldsymbol{\delta}\left(\boldsymbol{\varepsilon}+\mathbf{u}-\mathbf{x}_{\mathbf{i}}\right)$

Integrating (5) over $\mathrm{u}$ in infinite limits, taking into account the distribution density $\boldsymbol{\omega}_{\boldsymbol{u}}$ (u), we obtain the unconditional distribution density of the digital measurement error: $\boldsymbol{\omega}_{\mathbf{u}}(\varepsilon)=\int_{-\infty}^{\infty} \omega_{\mathbf{u}}(\varepsilon \mid \mathbf{u}) \boldsymbol{\omega}_{\mathbf{u}}(\mathbf{u}) \mathbf{d u}$, т. е.

$$
\omega_{\mathbf{u}}(\varepsilon \mid \mathbf{u})=\sum_{\mathbf{i}} p_{\mathbf{i}}\left(x_{\mathbf{i}}-\varepsilon\right) \omega_{\mathbf{u}}\left(x_{\mathbf{i}}-\varepsilon\right) .
$$

\section{Findings}

From a comparison of expressions (3) and (5) for the conditional error of control and digital measurement and (4) and (6) for the unconditional, it follows that if the conditional probability of the control error $p_{-}$(i) (u) is specified, then the discrete the conditional distribution density of the error and digital measurement $\omega(\varepsilon \mid u)$ (if the nominal values $x \_i$ assigned to the quantization intervals are specified), and vice versa, setting the conditional distribution of the digital measurement error is equivalent to setting the conditional probabilities of control errors. However, there is no unambiguous relationship between the unconditional probability of control errors $p_{-} i k$ and the unconditional distribution density of the digital measurement error $\omega_{-} \mathrm{u}(\mathcal{E})$. For an exhaustive judgment about the probabilistic characteristics of a control error or digital measurement, it is enough to have the conditional distribution of the reduced comparison error and the distribution of the converted value [10].

Let us now consider a circuit that also contains a digital processing device UPC (Figure 1). Let the conditional error probability of converting an input analog signal $u$ into a digital signal z be characterized by a square matrix $\left\|\boldsymbol{p}_{j \boldsymbol{k}}\right\|$, and the error probability of digital processing, converting digital signal z into digital signal y, is a square matrix $\left\|\boldsymbol{p}_{i j}\right\|$. Then the probability matrix of the control error $\left\|\boldsymbol{p}_{i k}\right\|$ for the circuit as a whole is found by matrix multiplication

$$
\left\|p_{i k}\right\|=\left\|p_{i j}\right\|\left\|p_{j k}\right\|
$$

those. each element of the matrix

$\boldsymbol{p}_{i k}=\sum_{i} \boldsymbol{p}_{i j} \boldsymbol{p}_{j k}$.

Conditional probability of assigning a quantity и к $\boldsymbol{Q}_{\boldsymbol{i}}$ in this case is expressed as

$$
\boldsymbol{p}_{\boldsymbol{i}}(\boldsymbol{u})=\sum_{i} \boldsymbol{p}_{i j} \boldsymbol{p}_{j} .
$$

For digital measurement with digital processing we have:

$$
\begin{gathered}
\omega_{u}(\varepsilon \mid u)=\sum_{i} \sum_{j} p_{i j} p_{i}(u) \delta\left(\varepsilon+u-x_{i}\right) ; \\
\omega_{u}(\varepsilon)=\sum_{i} \sum_{j} p_{i j} p_{i}\left(x_{i}-\varepsilon\right) \omega_{u}\left(x_{i}-\varepsilon\right) .
\end{gathered}
$$

The general formulas obtained here form the basis, which we will use in the future in order to derive the probabilistic characteristics of errors of the main transformations and operations used in centralized control systems [11].

\section{Conclusion}

When analyzing the accuracy of operational control, an important role is also played by the fundamental features of the centralized control equipment itself [12]. In the centralized 
control equipment, as a rule, digital conversion and digital presentation of measurement results are provided; this means that the operation of obtaining a number expressing the measurement result is automated, as a result of which, on the one hand, subjective human errors are eliminated, and on the other hand, the possibility of introducing amendments into the result that are not provided for in advance by the design or program of the device is excluded [13]. Formally, the result of measurement by which we judge its accuracy, in centralized control systems, should be considered the result produced by the equipment in digital form, and not the same result with a correction made by a person, even if he knows the necessary correction [14]. Note that due to the large number of controlled quantities, there is no possibility to make the necessary amendment, not only formally, but usually also in essence.

Another feature of the analysis of the accuracy of centralized control is determined by the principle of running around, on which the equipment is built . Repeated use of the same devices and their units for homogeneous processing of a large number of signals makes it difficult to introduce the necessary automatic correction. In addition, the round-trip introduces an additional error that must be taken into account along with dynamic errors of a different origin. In sweeping systems, the current result of a control or measurement operation usually has to be considered the last result, which changes abruptly only after a new operation is performed in the next sweep cycle.

The characteristics that need to be obtained as a result of measurement or control operations do not exist for one point in time, but in principle can be determined only over a certain period of time. Such characteristics are, in particular, the integral of the process time over a certain time interval and the average value of the value in the interval, as well as complex indicators that are a function of several processes and related to certain time intervals.

The aim of the article is to calculate the accuracy of centralized control systems. The accuracy of systems as a whole can be investigated not only by calculation, but also experimentally, and in this regard, with the conditions of use and a large number of controlled quantities, there are difficulties. In addition, experimental research is only possible for already developed, manufactured and operating systems. Even already manufactured systems, due to their complexity, are much more convenient to investigate in parts. The study of individual blocks also has certain advantages on existing systems, since during operation it is necessary to replace blocks, and, of course, it is important to be able to estimate the result of such a replacement in advance, instead of carrying out a cumbersome experimental study again after replacement.

Regarding the characteristics of random errors, which are determined experimentally, in the presentation it is assumed almost everywhere that such characteristics have already been found, and questions related to obtaining probabilistic characteristics from the experimental data are not subject to consideration.

This paper has been supported by the RUDN University Strategic Academic Leadership Program.

\section{References}

1. M. Andriluka, S. Roth, B. Schiele, Pictorial structures revisited: People detection and articulated pose estimation, Proceedings of IEEE Conference on Computer Vision and Pattern Recognition, 1014-1021 (Miami, FL, USA, June 2009)

2. B. Aryal Becerik-Gerber, Skin temperature extraction using facial landmark detection and thermal imaging for comfort assessment, In Proceedings of the 6th ACM International Conference on Systems for Energy-Efficient Buildings, Cities, and Transportation (BuildSys'19), 71-80 (New York, NY, USA, 13-14 November 2019) 
3. M. Burzo, M. Abouelenien, V. Pérez-Rosas, C. Wicaksono, Y. Tao, R. Mihalcea, Using infrared thermography and biosensors to detect thermal discomfort in a building's inhabitants, In Proceedings of the ASME 2014 International Mechanical Engineering Congress and Exposition, 1-11 (Montreal, Quebec, Canada, 14-20 November 2014)

4. Z. Cao, T. Simon, S. E. Wei, Y. Sheikh, Realtime multi-person $2 d$ pose estimation using part affinity fields, In Proceedings of the IEEE Conference on Computer Vision and Pattern Recognition (CVPR), 7291-7299 (Honolulu, HI, USA, 21-26 July 2017)

5. O. Çeliktutan, S. Ulukaya, B. Sankur, A comparative study of face landmarking techniques, EURASIP J. Image Video Process., 13 (2013)

6. Y. Chen, B. Lu, Y. Chen, X. Feng, Breathable and stretchable temperature sensors inspired by skin, Sci. Rep., 5, 11505 (2015)

7. X. Cheng, B. Yang, A. Hedman, T. Olofsson, H. Li, L.V. Gool, NIDL: A pilot study of contactless measurement of skin temperature for intelligent building, Energ. Build., 198, 340-352 (2019)

8. A. L. Chupin, O. A. Yurchenko, Zh. S. Lemesheva, A. Y. Pak, M. B. Khudzhatov, Development of Logistical Technologies in Management of Intellectual Transport Systems in the Russian Federation, Part of the Lecture Notes in Networks and Systems book series, LNNS, Digital Economy: Complexity and Variety vs. Rationality, 87, 778784

9. A. Ghahramani, G. Castro, B. Becerik-Gerber, X. Yu, Infrared thermography of human face for monitoring thermoregulation performance and estimating personal thermal comfort, Build. Environ., 109, 1-11 (2016)

10. A. Ghahramani, G. Castro, S. A. Karvigh, B. Becerik-Gerber, Towards unsupervised learning of thermal comfort using infrared thermography, Appl. Energ., 211, 41-49 (2018)

11. A. A. Gibadullin, J. A. Romanova, D. E. Morkovkin, R. M. Pirakov, Assessment of the level of environmental innovation in industrial production and information and communication sphere, Journal of Physics: Conference Series, 1679(5), 052076 (2020)

12. M. R. Kurbonzoda, A. A. Gibadullin, D. E. Morkovkin, J. N. Nesterenko, Assessment of factors hindering innovative development, Journal of Physics: Conference Series, 1679(3), 032065 (2020)

13. A. Mikhaylov, N. Moiseev, K. Aleshin, T. Burkhardt, Global climate change and greenhouse effect. Entrepreneurship and Sustainability Issues, 7(4), 2897-2913 (2020)

14. D. Morkovkin, D. Lopatkin, M. Sadriddinov, T. Shushunova, A. Gibadullin, O. Golikova, Assessment of innovation activity in the countries of the world, E3S Web of Conferences, 157, 04015 (2020) 